\title{
Chaotic Motion in Velocity Control System of Induction Motor
}

\author{
Toshiyuki ASAKURA \\ Faculty of Engineering, Fukui University, 3-9-1 Bunkyo, 910-8507 Fukui, Japan \\ asakura@scmc.mech.fukui-u.ac.jp \\ Keizo YONEDA \\ Faculty of Engineering, Fukui University, 3-9-1 Bunkyo, 910-8507 Fukui, Japan \\ yoneda@scmc.mech.fukui-u.ac.jp
}

\begin{abstract}
This paper is concerned with the occurrence of chaotic motion in velocity control system of induction motor. Though the control of induction motor is very difficult, it is currently used by realization of so-called vector control method. The reason is that the induction motor is superior to a dc motor in maintenance. But, the stability of the velocity control system is not established, neither is robustness. The dynamics of the induction motor is represented by nonlinear equations with three inputs and three outputs. Though precise parameters are necessary for the vector control method, it is difficult to obtain the precise values. Therefore, there are often identified errors in parameters and then the effect of the nonlinear terms remains. On this occasion, it is known that chaotic motions may occur in the system. In this paper, the existence of chaotic motion in such system can be verified using the concept of Lyapunov exponent, and the generating conditions of chaotic motion are found, when the desired value and load conditions are given.
\end{abstract}

\section{Introduction}

The induction motor is superior to a dc motor in maintenance. The control method which is widely used for the induction motor is a vector control. (1)-(3) Though the precise parameter values are necessary for a vector control, it is generally difficult to identify these parameters. The existence of identification errors for parameters disturbs linearization in the vector control and the effect of nonlinear terms remains. Then, it may be thought that chaotic motion occurs. The induction motor is driving source with nonlinear characteristics. Many reports of $\operatorname{chaos}^{(4)-(8)}$ occurring in mechanical systems concern with external force, for example, such as a pendulum or a nonlinear restoring force. In this case, it's presumed that the driving source has a constant torque. Then, there are few reports with respect to the occurrence of chaos based on the effect of nonlinear characteristics in the driving source. ${ }^{(9)(10)}$ In this research, the dynamics of the induction motor is treated considering driving source, in which the velocity control system is composed using vector control method. At this time, the responses are examined in cases of periodic input and step signal with periodic input, and the occurrence of chaotic motion verified using Lyapunov exponent. Also, in these cases, the generating conditions of chaos are shown for parameters of desired value and load conditions.

\section{Modeling of Induction Motor \\ 2.1 Circuit Equation of Induction Motor}

Symbols used in this paper are denoted below.

$i$ : stator current, $\Phi$ : rotor flux,

$\omega_{r}$ : rotor angular velocity, $v$ : stator voltage,

$\omega_{e}$ : electrical angular velocity, $R$ : resistance,

$L$ : coil inductance, $M$ : mutual inductance, $J$ : inertia,

$p$ : number of pole pairs, $D$ : viscosity constant

$\sigma=1-M^{2} / L_{s} L_{r}:$ leakage constant,

$T_{r}=L_{r} / R_{r}$ : rotor time constant

The block diagram of velocity control system is shown in Fig.1. The circuit equations of induction motor are given as,

$i_{\gamma s}=-\left(\frac{R_{s}}{\sigma L_{s}}+\frac{1-\sigma}{\sigma T_{r}}\right) i_{\gamma}+\omega_{e} i_{\delta s}+\frac{M}{\sigma L_{s} L_{r}} \frac{1}{T_{r}} \Phi_{\gamma r}+\frac{M}{\sigma L_{s} L_{r}} \omega_{r} \Phi_{\delta r}+\frac{v_{\gamma s}}{\sigma L_{s}}$

$i_{\delta s}=-\omega_{e} i_{\gamma s}-\left(\frac{R_{s}}{\sigma L_{s}}+\frac{1-\sigma}{\sigma T_{r}}\right) i_{\delta s}-\frac{M}{\sigma L_{s} L_{r}} \omega_{r} \Phi_{r r}+\frac{M}{\sigma L_{s} L_{r}} \frac{1}{T_{r}} \Phi_{s}+\frac{v_{\delta s}}{\sigma L_{s}}$

$\dot{\Phi}_{r r}=\frac{M}{T_{r}} i_{r s}-\frac{1}{T_{r}} \Phi_{r r}+\left(\omega_{e}-\omega_{r}\right) \Phi_{\delta r}$

$\dot{\Phi}_{\delta r}=\frac{M}{T_{r}} i_{\delta}-\left(\omega_{e}-\omega_{r}\right) \Phi_{r r}-\frac{1}{T_{r}} \Phi_{\delta r}$

$\dot{\omega}_{r}=\frac{p M}{J L_{r}}\left(i_{\delta s} \Phi_{r r}-i_{r s} \Phi_{\delta r}\right)-\frac{D}{J} \omega_{r}$

where "॰" shows time differential.

In the construction of velocity control is shown of Fig.1, the parameter $M / T_{r}$ is an important factor for vector control of induction motor. As this parameter includes $R_{r}$ which changes under the influence of heat etc., it may be considered that an identification error occurs through the difference between the true value inside a motor and the control value. Letting this parameter error be set with $K_{e}$, the equations of velocity control are given as follows.

$$
\begin{aligned}
& i_{r s}=\frac{\Phi^{*}{ }_{r}}{M}-\Phi_{\delta r}\left\{G_{\omega}(s)\left(\omega^{*}{ }_{r}-\omega_{r}\right)\right\} \\
& i_{i s}=\frac{\Phi_{\delta r}^{*}}{M}+\Phi_{r r}\left\{G_{\omega}(s)\left(\omega^{*},-\omega_{r}\right)\right\} \\
& v_{p s}=G_{\gamma}(s)\left(i^{\cdot}{ }_{i s}-i_{\gamma s}\right) \\
& v_{\delta s}=G_{\delta}(s)\left(i_{i s}^{*}-i_{\delta s}\right)
\end{aligned}
$$




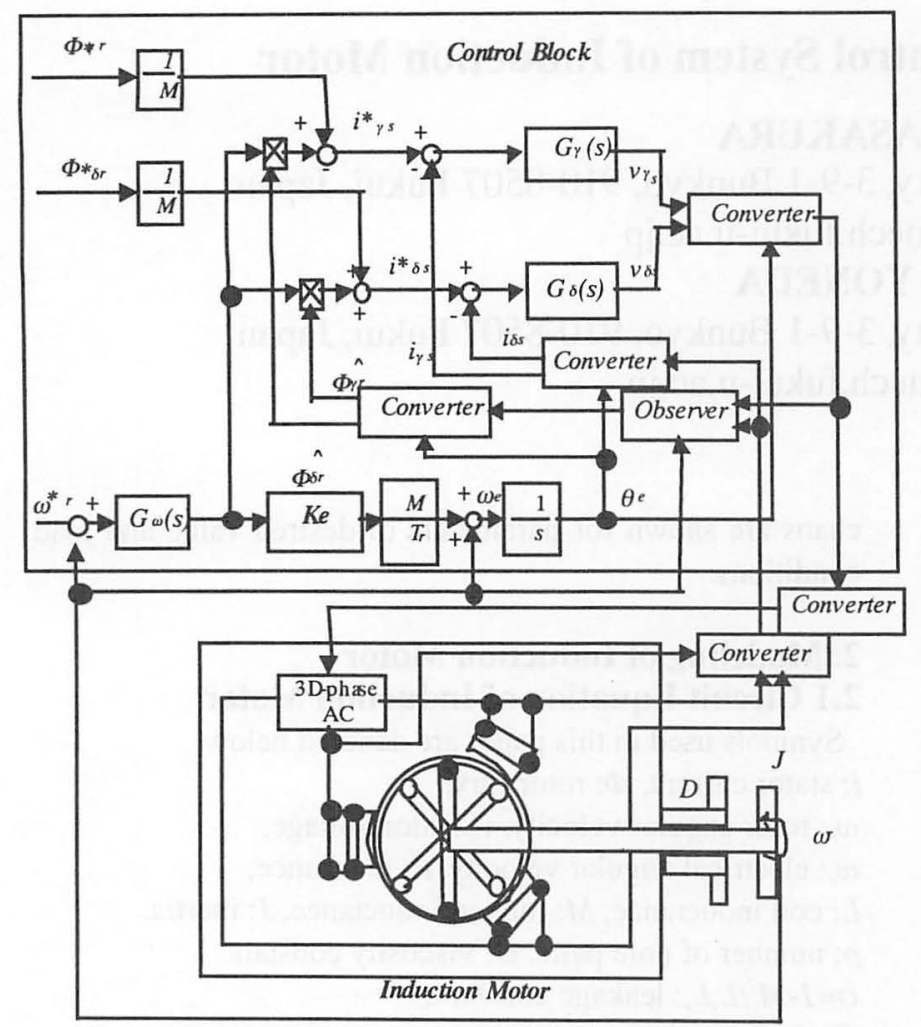

Fig.1 Block Diagram of Velocity Control System

$\omega_{e}-\omega_{r}=K_{e} \frac{M}{T_{r}}\left\{G_{\omega}(s)\left(\omega^{*},-\omega_{r}\right)\right\}$

where "*" shows desired value.

$G_{r}(s), G_{\delta}(s)$ and $G_{\omega}(s)$ are PI controller, with integral time $T_{i}$ and proportional gain $K$, as follows.

$G(s)=K\left(1+\frac{1}{T_{i} s}\right)$

\subsection{Observer}

Though the values $\Phi_{p}$ and $\Phi_{\delta}$ are required to perform vector control, it's difficult to measure directly. Then, the observer is composed. In order to make a setup of observer gain easy, the fixed $\mathrm{d}-\mathrm{q}$ coordinate is considered. The equations of observer are as follows.

$\dot{\hat{x}}=\left[\begin{array}{ll}a_{11} & a_{12} \\ a_{21} & a_{22}\end{array}\right] \hat{x}+\left[\begin{array}{c}b_{1} \\ 0\end{array}\right] u+\left[\begin{array}{l}0 \\ k_{1}\end{array}\right](y-\hat{y})$,

Here, the variables are,

$\hat{x}=\left[\begin{array}{llll}\hat{i}_{d s} & \hat{i}_{q s} & \hat{\Phi}_{d r} & \hat{\Phi}_{q r}\end{array}\right]^{r}, u=\left[\begin{array}{ll}v_{d s} & v_{q s}\end{array}\right]^{r}$

$y=\left[\begin{array}{ll}i_{d s} & i_{q s}\end{array}\right]^{T}$ and $\hat{y}=\left[\begin{array}{ll}\hat{i}_{d s} & \hat{i}_{q s}\end{array}\right]^{T}$

Also, each coefficient save given as,

$a_{11}=-\left(\frac{R_{s}}{\sigma L_{s}}+\frac{1-\sigma}{\sigma T_{r}}\right) I, a_{12}=\frac{M}{\sigma L_{s} L_{r}} \frac{1}{T_{r}} I+\frac{M}{\sigma L_{s} L_{r}} \omega_{r} J$,

$a_{21}=\frac{M}{T_{r}} I, a_{22}=-\frac{1}{T_{r}} I-\omega_{r} J, b_{1}=\frac{1}{\sigma L_{s}} I$

$k_{1}=\frac{M}{T_{r}} I, I=\left[\begin{array}{ll}1 & 0 \\ 0 & 1\end{array}\right]$ and $J=\left[\begin{array}{cc}0 & 1 \\ -1 & 0\end{array}\right]$,

where " $\wedge$ " is an estimated value and "T" a transpose. Also, the transform matrix $C_{1}$ from $\gamma-\delta$ to $\mathrm{d}$-q coordinate and $C_{2}$ from d-q to $\gamma-\delta$ coordinate are respectively, $C_{1}=\left[\begin{array}{cc}\cos \theta_{e} & -\sin \theta_{e} \\ \sin \theta_{e} & \cos \theta_{e}\end{array}\right]$ and $C_{2}=\left[\begin{array}{cc}\cos \theta_{e} & \sin \theta_{e} \\ -\sin \theta_{e} & \cos \theta_{e}\end{array}\right]$.

In this control system, first, Eqs.(6) to (10) are calculated by giving $\Phi_{r}^{*}{ }_{r} \Phi^{*}{ }_{\delta n}$ and $\omega^{*}{ }_{r}$ as desired values. At result, $v_{\gamma s}$ and $v_{\delta s}$ are obtained as outputs. Also, $\omega_{e}$ is obtained by adding $\omega_{r}$ to $\omega_{e}-\omega_{r}$ Second, $v_{p} v_{\delta s}$ and $\omega_{e}$ are, as input values, given to the induction motor, and the outputs $i_{r,} i_{d s}$ and $\omega_{r}$ detected here are feedback to the control block. Finally, after coordinate conversion of $C_{l,}, v_{\gamma,}, v_{\delta s} i_{\gamma,}$ and $i_{\delta s}$ are transferred to the observer and then the observer outputs $\Phi_{d r}$ and $\Phi_{q r}$, after coordinate conversion of $C_{2}$, feedback to control block. The differential equations for this control system are given as Eqs.(13) (25).

$$
\begin{aligned}
x_{1}= & i_{r s}, \quad x_{2}=i_{\delta s}, \quad x_{3}=\Phi_{\gamma r}, \quad x_{4}=\Phi_{\delta r}, \quad x_{5}=\omega_{r}, \quad x_{6}=\theta_{e}, \quad x_{7}=\int\left(i_{\gamma s}^{*}-i_{\gamma s}\right) d t x_{8}=\int\left(i_{i s}-i_{\delta s}\right) d t \\
x_{9}= & \int\left(\omega^{*},-\omega_{r}\right) d t x_{10}=\hat{i}_{d s}, x_{11}=\hat{i}_{q s}, \quad x_{12}=\hat{\Phi}_{d r}, \quad x_{13}=\hat{\Phi}_{q r} \\
\dot{x}_{1}= & -\left(\frac{R_{s}}{\sigma L_{s}}+\frac{1-\sigma}{\sigma T_{r}}\right) x_{1}+\left\{x_{5}+K_{e} K_{\omega} \frac{M}{T_{r}}\left(\omega^{\cdot}{ }_{r}-x_{5}+\frac{x_{9}}{T_{i \omega}}\right)\right\} x_{2}+\frac{M}{\sigma L_{s} L_{r}} \frac{1}{T_{r}} x_{3}+\frac{M}{\sigma L_{s} L_{r}} x_{4} x_{5} \\
& +\frac{K_{\gamma}}{\sigma L_{s}}\left\{\frac{\Phi^{*}{ }_{r r}}{M}-K_{\omega}\left(\omega^{*}{ }_{r}-x_{5}+\frac{x_{9}}{T_{i \omega}}\right)\left(-x_{12} \sin x_{6}+x_{13} \cos x_{6}\right)-x_{1}+\frac{x_{7}}{T_{i \gamma}}\right\} \\
\dot{x}_{2}= & -\left(\frac{R_{s}}{\sigma L_{s}}+\frac{1-\sigma}{\sigma T_{r}}\right) x_{2}-\left\{x_{5}+K_{e} K_{\omega} \frac{M}{T_{r}}\left(\omega^{*}{ }_{r}-x_{5}+\frac{x_{9}}{T_{i \omega}}\right)\right\} x_{1}+\frac{M}{\sigma L_{s} L_{r}} \frac{1}{T_{r}} x_{4}-\frac{M}{\sigma L_{s} L_{r}} x_{3} x_{5} \\
& +\frac{K_{\delta}}{\sigma L_{s}}\left\{\frac{\Phi^{*}}{M}-K_{\omega}\left(\omega_{r}^{*}-x_{5}+\frac{x_{9}}{T_{i \omega}}\right)\left(x_{12} \cos x_{6}+x_{13} \sin x_{6}\right)-x_{2}+\frac{x_{8}}{T_{i \delta}}\right\}
\end{aligned}
$$




$$
\begin{aligned}
& \dot{x}_{3}=\frac{M}{T_{r}} x_{1}-\frac{1}{T_{r}} x_{3}+K_{e} K_{\omega} \frac{M}{T_{r}}\left(\omega^{*}{ }_{r}-x_{5}+\frac{x_{9}}{T_{i \omega}}\right) x_{4} \\
& \dot{x}_{4}=\frac{M}{T_{r}} x_{2}-K_{e} K_{\omega} \frac{M}{T_{r}}\left(\omega^{*}{ }_{r}-x_{5}+\frac{x_{9}}{T_{i \omega}}\right) x_{3}-\frac{1}{T_{r}} x_{4} \\
& \dot{x}_{5}=\frac{p M}{J L_{r}}\left(x_{2} x_{3}-x_{1} x_{4}\right)-\frac{D}{J} x_{5} \\
& \dot{x}_{6}=x_{5}+K_{e} K_{\omega} \frac{M}{T_{r}}\left(\omega_{r},-x_{5}+\frac{x_{9}}{T_{i \omega}}\right) \\
& \dot{x}_{7}=\frac{\Phi^{\cdot}{ }_{r r}}{M}-K_{\omega}\left(\omega^{*}{ }_{r}-x_{5}+\frac{x_{9}}{T_{i \omega}}\right)\left(-x_{12} \sin x_{6}+x_{13} \cos x_{6}\right)-x_{1} \\
& \dot{x}_{8}=\frac{\Phi_{\delta}^{*}}{M}+K_{\omega}\left(\omega_{r}-x_{5}+\frac{x_{9}}{T_{i \omega}}\right)\left(x_{12} \cos x_{6}+x_{13} \sin x_{6}\right)-x_{2} \\
& \dot{x}_{9}=\omega^{*},-x_{5} \\
& \dot{x}_{10}=-\left(\frac{R_{s}}{\sigma L_{s}}+\frac{1-\sigma}{\sigma T_{r}}\right) x_{10}+\frac{M}{\sigma L_{s} L_{r}} \frac{1}{T_{r}} x_{12}+\frac{M}{\sigma L_{s} L_{r}} x_{5} x_{13} \\
& +\frac{K_{\gamma}}{\sigma L_{s}}\left\{\frac{\Phi^{*}{ }_{i r}}{M}-K_{\omega}\left(\omega^{*}-x_{5}+\frac{x_{9}}{T_{i \omega}}\right)\left(-x_{12} \sin x_{6}+x_{13} \cos x_{6}\right)-x_{1}+\frac{x_{7}}{T_{i \gamma}}\right\} \cos x_{6} \\
& -\frac{K_{\delta}}{\sigma L_{s}}\left\{\frac{\Phi_{\delta}^{*}}{M}-K_{\omega}\left(\omega^{*}{ }_{r}-x_{5}+\frac{x_{9}}{T_{i \omega}}\right)\left(x_{12} \cos x_{6}+x_{13} \sin x_{6}\right)-x_{2}+\frac{x_{8}}{T_{i \delta}}\right\} \sin x_{6} \\
& \dot{x}_{11}=-\left(\frac{R_{s}}{\sigma L_{s}}+\frac{1-\sigma}{\sigma T_{r}}\right) x_{11}-\frac{M}{\sigma L_{s} L_{r}} x_{5} x_{12}+\frac{M}{\sigma L_{s} L_{r}} \frac{1}{T_{r}} x_{13} \\
& +\frac{K_{\gamma}}{\sigma L_{s}}\left\{\frac{\Phi^{\cdot}{ }_{i r}}{M}-K_{\omega}\left(\omega^{\cdot}{ }_{r}-x_{5}+\frac{x_{9}}{T_{i \omega}}\right)\left(-x_{12} \sin x_{6}+x_{13} \cos x_{6}\right)-x_{1}+\frac{x_{7}}{T_{i \gamma}}\right\} \sin x_{6} \\
& +\frac{K_{\delta}}{\sigma L_{s}}\left\{\frac{\Phi^{*}{ }_{\delta r}}{M}-K_{\omega}\left(\omega^{*}{ }_{r}-x_{5}+\frac{x_{9}}{T_{i \omega}}\right)\left(x_{12} \cos x_{6}+x_{13} \sin x_{6}\right)-x_{2}+\frac{x_{8}}{T_{i \delta}}\right\} \cos x_{6} \\
& \dot{x}_{12}=-\frac{1}{T_{r}} x_{12}-x_{5} x_{13}+\frac{M}{T_{r}}\left(x_{1} \cos x_{6}-x_{2} \sin x_{6}\right) \\
& \dot{x}_{13}=x_{5} x_{12}-\frac{1}{T_{r}} x_{13}+\frac{M}{T_{r}}\left(x_{1} \sin x_{6}+x_{2} \cos x_{6}\right)
\end{aligned}
$$

\section{Simulation}

Parameters of velocity control are given in Table 1. Also, $\omega^{*}$ is given as follows.

$\omega^{*}{ }_{r}=U+\Omega \cos 2 \pi f t$

This equation means the case of step input with a periodic signal. Numerical calculations are performed by RungeKutta method, in which a sampling time is $0.001 \mathrm{~s}$ and all initial values are set as 0 . Identification error $K_{e}$, parameters of $\omega_{n}^{*} U, \Omega, f$, and load parameters $D, J$ are set arbitrarily.

\subsection{Calculation of Lyapunov Exponent}

The calculation procedure of Lyapunov exponent is as follows. As there are many variables, an approximated method is used. ${ }^{(11)}$ The solution of differential equations (13) (25) are expressed by vector $\boldsymbol{x}$ in 13-dimensional phase space. These differential equations are transformed to difference equations. The orbits of solution are given as $x_{n} \eta_{n}^{l} \eta_{n}^{2}$, if the initial condition are set as $x_{0} x_{0}+e^{1}$ and $x_{0}+e^{2}$. As a sampling time $\left\|e^{1}\right\|$ and $\left\|e^{2}\right\|$ is very small, the deviations $\varepsilon_{n}^{l}$ and $\varepsilon_{n}^{2}$ between $x_{n}$ and $\eta_{n}^{1}, x_{n}$ and $\eta_{n}^{2}$ are given respectively as,

$$
\begin{gathered}
\varepsilon^{1}{ }_{n}=\eta^{1}{ }_{n+1}-x_{n+1} \\
\varepsilon_{n}{ }_{n}=\eta^{2}{ }_{n+1}-x_{n+1}
\end{gathered}
$$

Accordingly, Lyapunov exponents $\lambda_{1}$ and $\lambda_{2}$ are defined by 


$$
\begin{aligned}
& \lambda_{1}=\lim _{n \rightarrow \infty} \frac{1}{n \Delta t} \sum_{j=0}^{n-1} \log \frac{\left\|\varepsilon^{1}{ }_{j}\right\|}{\left\|e^{1}\right\|} \\
& \lambda_{2}=\lim _{n \rightarrow \infty} \frac{1}{n \Delta t} \sum_{j=0}^{n-1} \log \frac{\left\|\varepsilon^{1}{ }_{j} \times \varepsilon^{2}{ }_{j}\right\|}{\left\|e^{1} \times e^{2}\right\|}-\lim _{n \rightarrow \infty} \frac{1}{n \Delta t} \sum_{j=0}^{n-1} \log \frac{\left\|\varepsilon^{1}{ }_{j}\right\|}{\left\|e^{1}\right\|}
\end{aligned}
$$

Through the calculations of Eqs. (29) and (30), it may be judged as chaos, when the maximum Lyapunov exponent is positive.

\subsection{Chaos by Periodic Input}

First, the desired input of Eq. (26) is set as $U=0, \Omega=20.0$ and $f=12.0$. Also, the load parameters are $J=0.0050$ and $D=0.0016$. According to the change of $K_{e}$, the bifurcation map is shown in Fig.2 and Lyapunov exponent in Fig.3. Lyapunov exponent is the quantity that the two close orbits leave with time in phase space. When this exponent is positive, it is judged to be chaos. From Fig.2, the bifurcation map $\omega_{r}$ shows the complicated branches after $K_{e}=1.25$ and then from Fig.3, Lyapunov exponent is positive in the range of complicated branches. Then, the chaos is well judged from Lyapunov exponent of Fig.3.

\section{Table 1 Motor Constants and Control Parameters}

\begin{tabular}{|lr|lr|}
\hline$R_{s}$ & $0.5 \Omega$ & $p$ & 2 \\
$R_{r}$ & $1.0 \Omega$ & $K_{\gamma}$ & $10.0 \Omega$ \\
$L_{s}$ & $0.105 \mathrm{H}$ & $T_{i \gamma}$ & $0.01 \mathrm{~s}$ \\
$L_{r}$ & $0.105 \mathrm{H}$ & $K_{\delta}$ & $10.0 \Omega$ \\
$M$ & $0.100 \mathrm{H}$ & $T_{i \delta}$ & $0.01 \mathrm{~s}$ \\
$\Phi^{*}{ }_{{ } r}$ & $0.25 \mathrm{Vs}$ & $K_{\omega}$ & $15.0 \Omega$ \\
$\Phi_{\delta \gamma}^{*}$ & $0.0 \mathrm{Vs}$ & $T_{i \omega}$ & $0.05 \mathrm{~s}$ \\
\hline
\end{tabular}

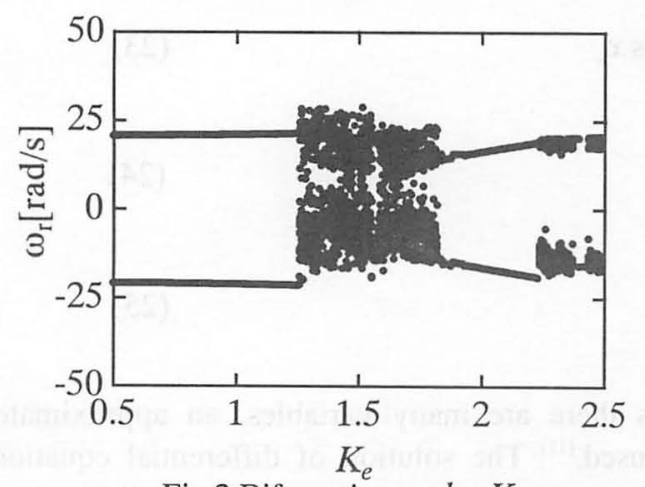

Fig.2 Bifurcation $\omega_{r}$ by $K_{e}$

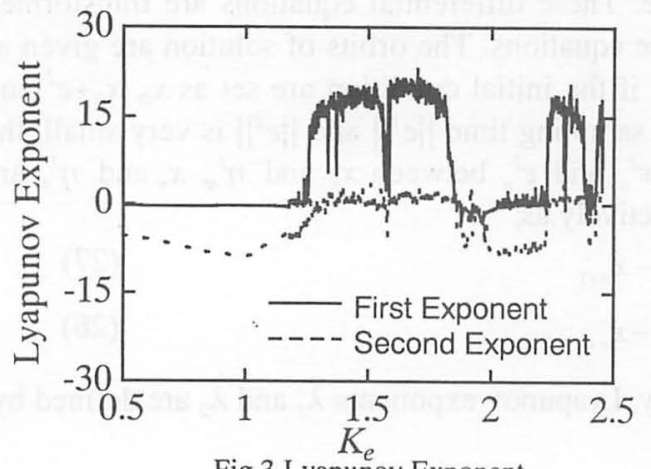

Fig.3 Lyapunov Exponent
Next, the generating conditions are considered, when the identification error is $K_{e}=1.30$. Figures 4 and 5 show the maps of behaviors of $\omega_{r}$ by the parameter variation of both desired value and load, respectively. Fig. 4 shows the range of chaotic behaviors by the variations of $\Omega$ and $f$, when $J=0.0050$ and $D=0.0016$. Similarly, Fig. 5 shows the range of chaos based on the variation of $J$ and $D$, when $\Omega=20.0$ and $f=12.0$.

From these figures, the generating conditions of chaos become obvious. In Fig.4, chaos occurs in the range of $\Omega=17.8 \sim 30.0, f=5.0 \sim 17.5$, and periodic solution exists in the range of $f=17.5 \sim 25.0$ close to this chaos range. In Fig.5, chaos solution exists in the range of $J=0.004 \sim 0.025$, $D=0.0 \sim 0.11$, and 1-cycle solution appears into the lower right of Fig.5. Fig. 6 shows the bifurcation map of $\omega_{r}$ by the

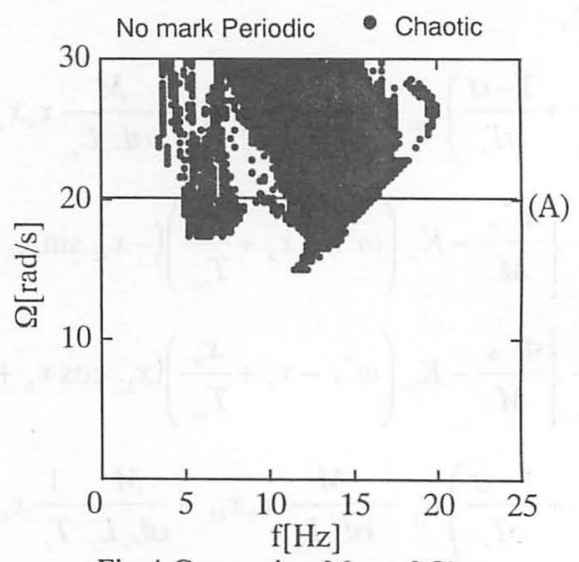

Fig.4 Generating Map of Chaos (by the variation of $\Omega$ and $f$ )

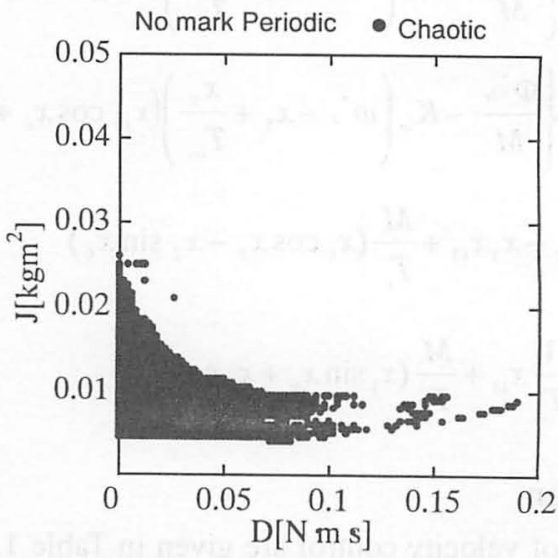

Fig.5 Generating Map of Chaos

(by the variation of $J$ and $D$ )

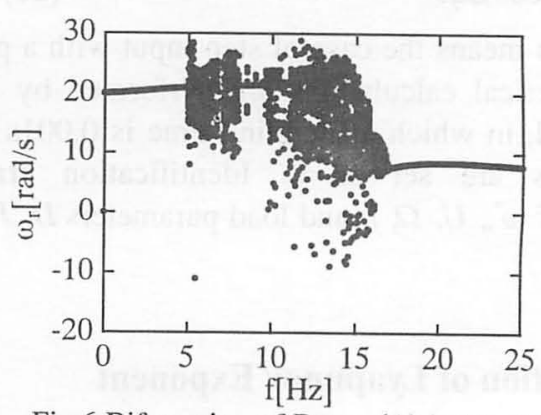

Fig.6 Bifurcation of Route (A) in Fig.4 
variation of $f$, when $\Omega=20.0$. After chaos generates at $f=5.0$, 1 -cycle solution and the chaos are mutually repeated. After periodic solution ends at $f=16.0$, it appears 1 -cycle solution. From Figure 4 and 5, it is known that the behaviors of $\omega_{r}$ change by parameter variation and chaotic motion appears, as mentioned above.

\subsection{Chaos by Step Input with Periodic Signal}

From the practical viewpoint, we'll consider the behavior with step input $U=75.0$ and periodic signal with $\Omega=15.0$, $f=12.0$. In Fig.7, the bifurcation map with maximum and minimum value of $\omega_{r}$ is shown by changing $K_{e}$. Also, Lyapunov exponent is drawn at the same time in Fig.8. The behavior becomes unstable more than $K_{e}=1.84$. The bifurcation map and Lyapunov exponent also correspond well. Comparing with the case of periodic case, it is remarkable point that an unstable solution appears by the addition of step input.

When $K_{e}=1.30$ is chosen in the range of chaos, the map of classified behavior $\omega_{r}$ is shown in Fig.9 by changing parameters of load. It's set with $\Omega=15.0, f=12.0$. Fig.9 is different from the case of periodic input. The range of chaos exits widely, and each range of chaos, 2-cycle and 1cycle solution exist in the lower right of this map. The bifurcation map of $\omega_{r}$ is shown in Fig. 10 where $J=0.0050$. After it changes to chaos suddenly at $D=0.028$, it becomes 2 -cycle. It changes to chaos again and 5-cycle window exists close to $D=0.12$ and 3 -cycle window close to $D=0.13$. Finally, to observe the chaotic behavior, an example of both the time response and Poincarè map are shown in Fig. 11.

The parameters are chosen as $U=0.0, \Omega=20.0, f=14.0$, $J=0.0050, D=0.0016$. It's judged that this angular velocity

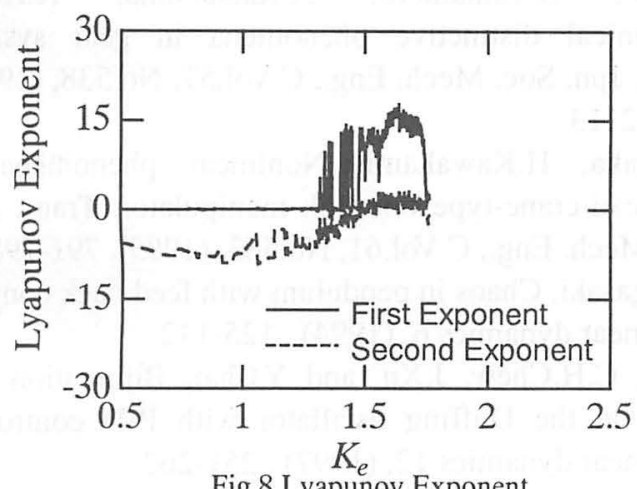

Fig.8 Lyapunov Exponent

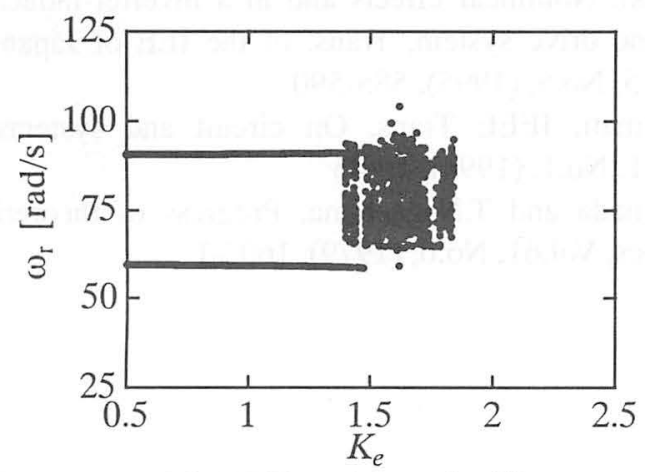

Fig.7 Bifurcation $\omega_{r}$ by $K_{e}$

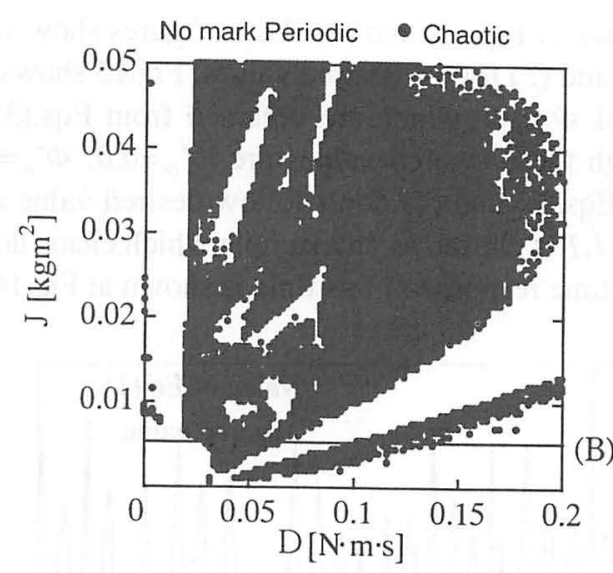

Fig.9 Classification of Response

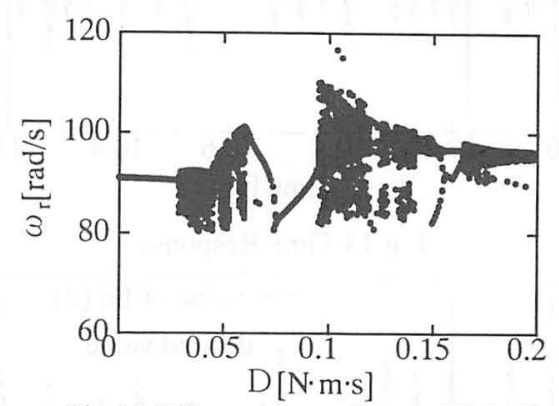

Fig.10 Bifurcation on Route (B) in Fig.9
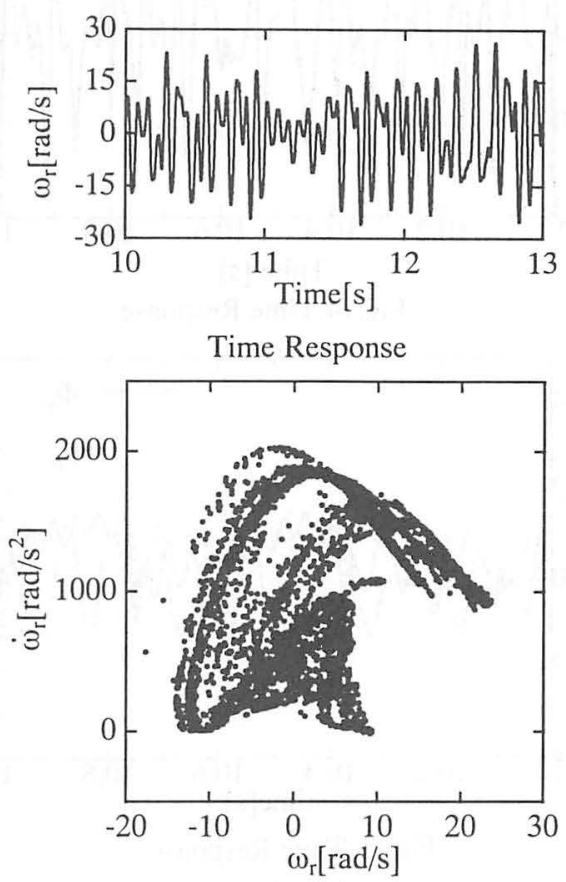

Poincare Map

Fig.11 Time Response and Poincare Map $\omega_{r}$ is chaos from time response of angular velocity and Poincarè map distribution.

\section{Analysis When Cause of Chaos Occurrence}

It's considered that chaotic behavior generates because there is something causes at variables. Then, we examined variables which are obtained from Eqs.(1) (4). The results are shown in Fig.13 Fig.14. Here, $U=0, \Omega=20.0, f=12.0$, $J=0.0050, D=0.0016$ and $K_{e}=1.30$.

Figure 13 and 14 show comparison of desired values of $i_{\delta s}$, 
$i_{r s}$ and values of Eqs.(1) and (2). These figures show values of Eqs.(1) and (2) follow desired values. Fig.15 shows time response of $\Phi_{\delta r}, \Phi_{\gamma r}$ which are obtained from Eqs.(3) and (4). Though these desired values are $\Phi^{*}{ }_{\delta r}=0.0, \Phi_{r r}^{*}=0.25$, values of Eqs.(3) and (4) don't follow desired value at all. When $K_{e}=1.1$ is chosen as an example which chaos doesn't occur, the time response at this time is shown at Fig.16.

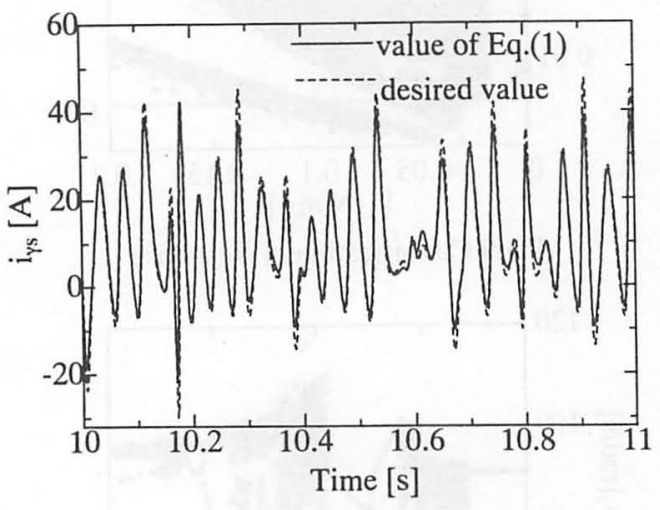

Fig.13 Time Response

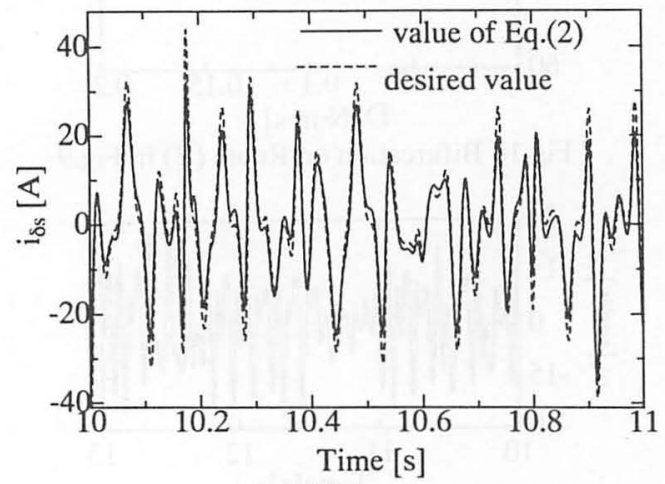

Fig.14 Time Response

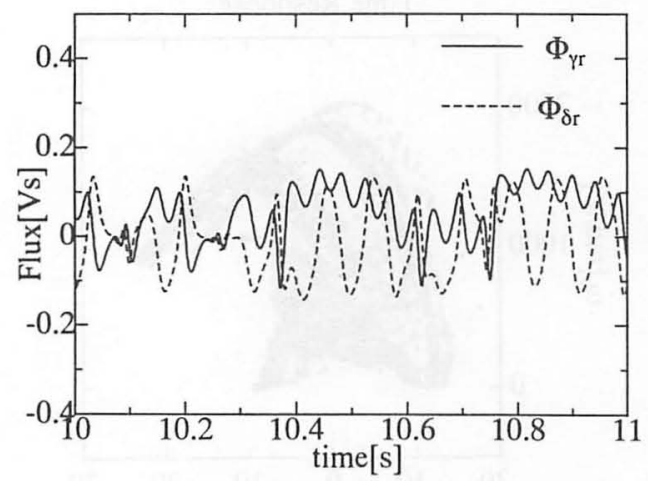

Fig.15 Time Response

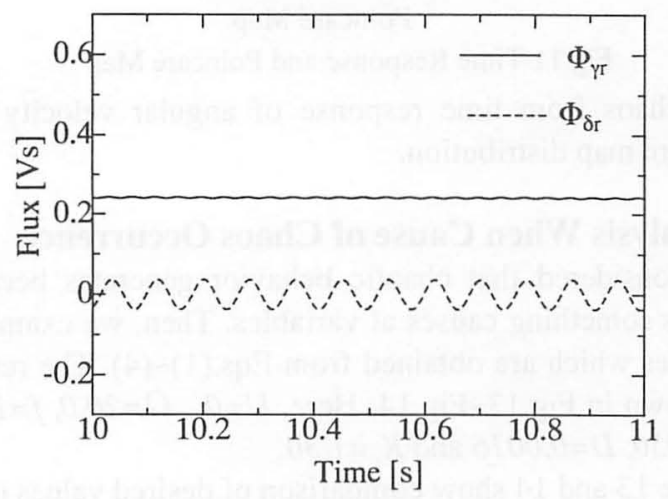

Fig.16 Time Response
The mentioned above, it's verified fluxes may not follow these desired values when chaos occurs in this system.

\section{Conclusion}

In this research, the analysis has been studied on the chaotic motion of induction motor when identification error exists. Consequently, the following results are verified.

1. The existence of chaotic motion has been clarified using Lyapunov exponent.

2. Based on the parameter change of both desired input and load, the solution behaviors have been classified and generating conditions of chaos found.

3. It's verified fluxes may not follow these desired values when chaos occurs in this system.

It is considered first and second analyses the mentioned above are useful to prevent occurrence chaos in this system. The third analysis is left to future study why fluxes don't follow these desired values when chaos occurs.

\section{Reference}

(1) Nakano, Vector control of AC motor, The nikkan kogyo shimbun LTD, Japan (1996), 1-33

(2) S.Shinnaka, Measurement and control, Vol.35, No.10 Jpn. (1996), 783-797

(3) N.Morishima, H.Maeda, S.Kodama, A robust control of induction motor drives by nonlinear state feedback, Vol.3, No.2, The society of instrument and control engineers, Japan (1990), 29-37

(4) Z.Xu, H.Tamura, Simulation of chaotic vibration of single-degree of-freedom magnetic levitation system, Trans. Jpn. Soc. Mech. Eng., C, Vol.61, No.583, (1995), 87-94

(5) K.Sato, S.Yamamoto, A.Katsunuma, K.Kono, Dynamical distinctive phenomena in gear system, Trans. Jpn. Soc. Mech. Eng., C Vol.57, No.538, (1991), 2108-2113

(6) Y.Tanaka, H.Kawakami, Nonlinear phenomena in overhead-crane-type one-link manipulator, Trans. Jpn. Soc. Mech. Eng., C Vol.61, No.583, (1995), 791-798

(7) K.Yagasaki, Chaos in pendulum with feed back control, Nonlinear dynamics 6, (1994), 125-142

(8) F.Cui, C.H.Chew, J.Xu, and Y.Chai, Bifurcation and chaos in the Duffing oscillator with PID controller, Nonlinear dynamics 12, (1997), 251-262

(9) S.Ozaki, Nonlinear effects and in a inverter-induction machine drive system, Trans. of the IEE of Japan, D Vol.115, No.5, (1995), 585-590

(10) N.Hemati, IEEE Trans. On circuit and systems I, Vol.41, No.1, (1994), 40-45

(11)I.Shimada and T.Nagashima, Progress of theoretical physics, Vol.61, No.6, (1979), 1605-1 